ITP.SB-93-22

\title{
High-Order Adiabatic Approximation for Non-Hermitian Quantum System and Complexization of Berry's Phase
}

\author{
Chang-Pu Sun \\ Physics Dpartment, Northeast Normal University, Changchun 130024, \\ P.R.China \\ and \\ Institute for Theoretical Physics, State University of New York, Stony \\ Brook, NY 11794-3840, USA
}

\begin{abstract}
In this paper the evolution of a quantum system drived by a non-Hermitian Hamiltonian depending on slowly-changing parameters is studied by building an universal high-order adiabatic approximation(HOAA) method with Berry's phase, which is valid for either the Hermitian or the non-Hermitian cases. This method can be regarded as a non-trivial generalization of the HOAA method for closed quantum system presented by this author before. In a general situation, the probabilities of adiabatic decay and non-adiabatic transitions are explicitly obtained for the evolution of the non-Hermitian quantum system. It is also shown that the non-Hermitian analog of the Berry's phase factor for the non-Hermitian case just enjoys the holonomy structure of the dual linear bundle over the parameter manifold. The non-Hermitian evolution of the generalized forced harmonic oscillator is discussed as an illustrative examples.
\end{abstract}

${ }^{1}$ accepted for publication in Physica Scripta 


\section{Introduction}

Since Berry's phase factor(BPF) was discovered in evolution of the quantum system with adiabatically-changing parameters [1], a few methods studying nonadiabatic evolution of a quantum system driven by a Hermitian Hamiltonian depending on slowly (but not adiabatically)-changing parameters have been presented in connection with BPF's [2-5]. In these methods, the high-order adiabatic approximation (HOAA) method was proposed by this author for the first time [4] and has been used and developed for many case [6-12]. However, all of these studies has not been concerned with a kind of important quantum system, the quantum open system that possesses a non-Hermitian $(\mathrm{nH})$ Hamiltonian. This paper will be devoted to the generalization of the HOAA method for such $\mathrm{nH}$ quantum system.

In fact, though the Hamiltonian for a closed quantum system, which is usually considered as a basic object in quantum theory, must be a Hermitian operator, many theories, such as the Fock-Krylov theorem [13], show the probability to apply the nH Hamiltonian for those quantum phenomena with dissipation, decay and relaxation $[14,15]$. Recently, many practical problems including the multiphoton ionization, the supermode free-electron laser and the transverse mode propagation in optical

resonator [16-19] have been concerned with the use of $\mathrm{nH}$ Schrodinger equation and $\mathrm{nH}$ Hamiltonian correspondingly. Since the occurrence of a BPF or its analog may be established when something in the considered system is varied, it is natural to generalize the concept of BPF for $\mathrm{nH}$ quantum system. More recently, some authors made this generalization and applied it to concrete physical problems [20-21], but their studies were only focused on the adiabatic case that the parameters change so slowly that transition between any two (quasi-) energy levels do not happen. In this paper we especially emphasized the non-adiabatic evolution of the $\mathrm{nH}$ quantum system and the geometry of the $\mathrm{nH}$ analog of BPF.

The paper is arranged as follows. In section 2, using a similarity transformation of the $\mathrm{nH}$ Hamiltonian, we build an universal formalism of the HOAA method for the $\mathrm{nH}$ quantum system. It is also available to the Hermitian quantum system. In 
section 3, we explicitly analyse the conditions under which the lowest order approximation,namely the the adiabatic approximation,can work well. We also compare our results with that obtained with the biorthonormal state method [20-21] in adiabatic case. In section 4 , we apply the general result to a simple toy model - the $\mathrm{nH}$ forced oscillator to show the usefulness of our analysis. In section 5 , we show that the nH analog of BPF appearing in an adiabatic evolution of $\mathrm{nH}$ quantum system are nonunitary holonomy group element for the dual bundles over the parameter maniford. In the further studies, we will provide more applications of this generalized HOAA method to some physical problems.

\section{Generalized HOAA Method for nH Quantum System}

Let us begin by setting some notations. The Hamiltonian of the quantum open system we consider as fillows is a $\mathrm{nH}$ operator

$$
\mathbf{H}=\mathbf{H}(t)=\mathbf{H}[\mathbf{R}]=\mathbf{H}[\mathbf{R}(t)]
$$

that depends on a set of slowly-changing parameters

$$
\mathbf{R}=\mathbf{R}(t):\left(R_{1}(t), R_{2}(t), \ldots, R_{L}(t)\right)
$$

We now assume that $\mathbf{H}(\mathrm{t})$ is diagonalizable at each instant $\mathrm{t}$, i.e., there exists a similarity transformation

$$
U(t)=U[\mathbf{R}]=U[\mathbf{R}(t)]
$$

such that

$$
U(t) \mathbf{H}(\mathbf{t}) U(t)^{-1}=H_{d}(t)=\left[\begin{array}{cccr}
\epsilon_{1}(t) & 0 & \ldots . & 0 \\
0 & \epsilon_{2}(t) & \ldots . & 0 \\
\ldots & \ldots & \ldots . & \ldots \\
0 & 0 & \ldots . . & \epsilon_{N}(t)
\end{array}\right]=\mathbf{H}_{d}(t)
$$

where the "quasi-energy levels"

$$
\epsilon_{k}(t)=\epsilon_{k}[\mathbf{R}], \quad k=1,2 \ldots, N
$$

may be complex and $\mathrm{U}(\mathrm{t})$ is not unitary correspondingly. 
Let

$$
|\Psi(t)>=U(t)| \Phi(t)>
$$

be a solution of the $\mathrm{nH}$ Schrodinger equation

$$
i \hbar \frac{\partial}{\partial t}|\Psi(t)>=\mathbf{H}(t)| \Psi(t)>\text {. }
$$

The equivalent wavefunction $\mid \Phi(t)>$ must satisfy an equivalent Schrodinger equation $(\mathrm{ESE})$

$$
i \hbar \frac{\partial}{\partial t}\left|\Phi(t)>=\mathbf{H}_{e}(t)\right| \Phi(t)>
$$

with the equivelent Hamiltonian

$$
\mathbf{H}_{e}(t)=\mathbf{H}_{d}(t)-i \hbar U(t)^{-1} \frac{\partial U(t)}{\partial t}
$$

Separate $\mathbf{H}_{e}(t)$ into two parts, the diagonal part

$$
\mathbf{H}_{0}(t)=\mathbf{H}_{d}(t)+\text { diagonal part of }\left[-i \hbar U(t)^{-1} \frac{\partial}{\partial t} U(t)\right]
$$

and the off-diagonal part

$$
\mathbf{V}(t)=\text { off diagonal part of }\left[-i \hbar U(t)^{-1} \frac{\partial}{\partial t} U(t)\right] .
$$

Then,

$$
\mathbf{H}_{e}(t)=\mathbf{H}_{0}(t)+\mathbf{V}(\mathbf{t})
$$

Late on we will show that the diagonal part $\mathbf{H}_{0}(t)$ governs an adiabatic evolution of the $\mathrm{nH}$ quantum system while the off-diagonal part $\mathbf{V}(t)$ governs the non-adiabatic transitions among the quasi-energy levels. In fact, since $\mathbf{V}(t)$ completely vanishes when $\mathbf{H}$ is independent of time $t$ and $\mathbf{H}=\mathbf{H}(t)=\mathbf{H}[\mathbf{R}]$ is a smooth function of $\mathbf{R}(t)$ , we can regard $\mathbf{V}(t)$ as a pertubation when $\mathbf{H}$ or $\mathbf{R}$ depends on time "slightly", i.e., $\mathbf{R}(t)$ changes as t slowly enough.

Based on the above decomposation of $\mathbf{H}_{e}(t)$, we can use the standard timedependent pertubation theory to solve the ESE (2.3), obtaining a approximate series solution

$$
|\Phi(t)>=| \Phi^{0}(t)>+\left|\Phi^{1}(t)>+\right| \Phi^{2}(t)>+\ldots+\mid \Phi^{l}(t)>+\ldots
$$


Here, the $l^{\prime}$ th order solution $\mid \Phi^{l}(t)>$ is determined by

$$
\begin{gathered}
i \hbar \frac{\partial}{\partial t}\left|\Phi^{0}(t)>=\mathbf{H}^{0}(t)\right| \Phi_{0}(t)>; \\
i \hbar \frac{\partial}{\partial t}\left|\Phi^{l}(t)>=\mathbf{H}_{\mathbf{0}}(t)\right| \Psi(t)>+\mathbf{V}(t) \mid \Phi^{l-1}(t)>. \\
l=1,2, \ldots,
\end{gathered}
$$

Because the above equations of $l^{\prime}$ th order solution $\mid \Phi^{l}(t)>$ only concern $(l-1)^{\prime}$ th order solutions $\mid \Phi^{l-1}(t)>$, we can get each order approximate solution starting from the zero'th order one

$$
\begin{gathered}
\left|\Phi^{0}(t)>=\exp \left[\frac{1}{i \hbar} \int_{0}^{t} \mathbf{H}_{0}\left(t^{\prime}\right) d t^{\prime}\right]\right| \Phi(0)> \\
\left|\Phi(0)>=U(0)^{-1}\right| \Psi(0)>.
\end{gathered}
$$

In order to write out thse solutions in a quite explicit form, we use the eigenstates

$$
\left|1>=\left[\begin{array}{l}
1 \\
0 \\
\cdot \\
0 \\
0
\end{array}\right], \quad\right| 2>=\left[\begin{array}{l}
0 \\
1 \\
\cdot \\
\cdot \\
0 \\
0
\end{array}\right], \ldots,\left|N-1>=\left[\begin{array}{l}
0 \\
0 \\
\cdot \\
\cdot \\
1 \\
0
\end{array}\right], \quad\right| N>=\left[\begin{array}{l}
0 \\
0 \\
\cdot \\
\cdot \\
0 \\
1
\end{array}\right]
$$

of $\mathbf{H}_{0}(t)$ with the corresponding eigenvalue

$$
E_{n}(t)=\epsilon_{n}(t)-\hbar \gamma_{n}(t)
$$

where the additional term to the quasi-energy

$$
\gamma_{n}(t)=i \int_{0}^{t}<n\left|U\left(t^{\prime}\right)^{-1} \frac{\partial U\left(t^{\prime}\right)}{\partial t^{\prime}}\right| n>d t^{\prime}
$$

will be proved to be the $\mathrm{nH}$ analog of Berry's phase. Then, each order solution

$$
\left|\Phi^{l}(t)>=\sum_{n=1}^{N} C_{n}^{l}(t) e^{i \gamma_{n}(t)} e^{\frac{1}{i \hbar} \int_{0}^{t} \epsilon_{n}\left(t^{\prime}\right) d t^{\prime}}\right| n>
$$

follows from eq.(2.6) immediately. Here, the coefficients $C_{n}^{l}(t)$ satisfy

$$
C_{n}^{0}(t)=<n|\Phi(0)>=<n| U(0)^{-1} \mid \Psi(0)>,
$$




$$
C_{n}^{l}(t)=\sum_{m=1}^{N} \frac{1}{i \hbar} \int_{0}^{t}<n\left|\mathbf{V}\left(t^{\prime}\right)\right| m>e^{i \int_{0}^{t^{\prime}} \omega_{n, m}(s) d s} C_{m}^{l-1}\left(t^{\prime}\right) d t^{\prime}
$$

where

$$
\omega_{n, m}=\frac{\epsilon_{n}(t)-\epsilon_{m}(t)+\dot{\gamma}_{m}(t)-\dot{\gamma}_{n}(t)}{\hbar}
$$

Notice that the difficulty that the eigenstates of $\mathbf{H}$ are not orthogonal to each other due to non-Hermiticity of $\mathbf{H}$ has been avoided in the above discussion by a trick building the ESE (2.3) to find the pertubation $\mathbf{V}(\mathrm{t})$. This is the key to our studies in this paper.

\section{Adiabatic Approximation and Comparision with Biorthonormal State}

\section{Method}

In this section our focus first is on the adiabatic conditions that the zero'th order (adiabatic) approximation can well approach the true evolution of the $\mathrm{nH}$ quantum system. Consider the integral

$$
\begin{gathered}
\mathbf{I}=\int_{0}^{t}<n\left|\mathbf{V}\left(t^{\prime}\right)\right| m>e^{i \int_{0}^{t^{\prime}} \omega_{n, m}(s) d s} d t^{\prime} \\
=\int_{0}^{t}<n\left|\mathbf{V}\left(t^{\prime}\right)\right| m>e^{-\operatorname{Im}\left[\int_{0}^{t^{\prime}} \omega_{n, m}(s) d s\right]} e^{i \operatorname{Re}\left[\int_{0}^{t^{\prime}} \omega_{n, m}(s) d s\right]} d t^{\prime}
\end{gathered}
$$

appearing in the first order approximation

$$
C_{n}^{1}(t)=\sum_{m=1}^{N} \frac{1}{i \hbar}<n\left|\Phi(0)>\int_{0}^{t^{\prime}}<n\right| \mathbf{V}\left(t^{\prime}\right) \mid m>e^{i \int_{0}^{t^{\prime}} \omega_{n, m}(s) d s} d t^{\prime} .
$$

Here, we have separated the damping factor

$$
e^{-\operatorname{Im}\left[\int_{0}^{t} \omega_{n, m}(s) d s\right]}
$$

and the oscillating factor

$$
e^{i R e\left[\int_{0}^{t} \omega_{n, m}(s) d s\right]} .
$$

If the latter oscillates so fast that the conditions

$$
\left|\frac{<n|\mathbf{V}(t)| m>e^{-\operatorname{Im}\left[\int_{0}^{t} \omega_{n, m}(s) d s\right]}}{\operatorname{Re}\left[\omega_{n, m}(t)\right] \hbar}\right| \ll 1
$$


hold for $m \neq n$, the integral $\mathbf{I}$ tends to zero. This statment can be proved by integrating $\mathbf{I}$ by part. Then, we get so-called adiabatic conditions (3.1). When they hold for a $\mathrm{nH}$ quantum system, we can ignore the higher-approximation solutions $\mid \Phi_{l}(t)>,(l=1,2, \ldots)$.

To compare our analysis with the biorthonormal state method in refs. $[20,21]$ in the adiabatic case, we define

$$
\begin{gathered}
\left|\phi_{n}(t)>=\right| \phi_{n}[\mathbf{R}]>=U(t) \mid n>, \\
\left|\chi_{n}(t)>=\right| \chi_{n}[\mathbf{R}]>=\left[U(t)^{-1}\right]^{\dagger} \mid n>,
\end{gathered}
$$

Obiviously, they are the eigenstates of $\mathbf{H}(t)$ and $\mathbf{H}(t)^{\dagger}$ respectively with the eigenvalues $\epsilon_{n}(t)$ and $\epsilon_{n}^{*}(t)$. Using the completeness relation and the orthonormal relations of states $\mid n>(n=1,2, \ldots, N)$ :

$$
\sum_{n=1}^{N}|n><n|=I(\text { unit operator }), \quad<n \mid m>=\delta_{m, n},
$$

we immmediately get the generalized completeness relations

$$
\sum_{n=1}^{N}\left|\chi_{n}(t)><\phi_{n}(t)\right|=\sum_{n=1}^{N}\left|\phi_{n}(t)><\chi_{n}(t)\right|=I
$$

and the biorthonomarl relations

$$
<\phi_{m}(t) \chi_{n}(t)>=<\chi_{m}(t) \mid \phi_{n}(t)>=\delta_{m, n} .
$$

In terms of the biorthonormal basis $\left\{\left|\phi_{n}(t)>,\right| \chi_{n}(t)>\right\}$, we reexpress the highorder approximations of the $\mathrm{nH}$ quantum evolution as

$$
\begin{aligned}
& \left|\Psi(t)>=\sum_{l=0}^{\infty}\right| \Psi^{l}(t)>=\sum_{l=0}^{\infty} U(t) \mid \Phi^{l}(t)> \\
& =\sum_{l=0}^{\infty} \sum_{n=1}^{N} C_{n}^{l}(t) e^{i \gamma_{n}(t)} e^{\frac{1}{i \hbar} \int_{0}^{t^{\prime}} \epsilon_{n}\left(t^{\prime}\right) d t^{\prime}} \mid \phi_{n}(t)>,
\end{aligned}
$$

with the coefficient equations:

$$
\begin{gathered}
C_{n}^{0}(t)=<\chi_{n}(0) \mid \Psi(0)> \\
C_{n}^{l}(t)=-\sum_{m=1}^{N} \frac{1}{i \hbar} \int_{0}^{t}<\chi_{n}\left(t^{\prime}\right) \mid \phi_{m}\left(t^{\prime}\right)>e^{i \int_{0}^{t^{\prime}} \omega_{n, m}(s) d s} C_{m}^{l-1}\left(t^{\prime}\right) d t^{\prime}
\end{gathered}
$$


for $l=1,2,3, \ldots$ Here, the $\mathrm{nH}$ analog of Berry's phase is rewritten as

$$
\gamma_{n}(t)=i \int_{0}^{t}<\chi_{n}\left(t^{\prime}\right) \mid \frac{d}{d t} \phi_{n}\left(t^{\prime}\right)>d t^{\prime}
$$

Correspondingly, the adiabatic conditions (3.1) are rewritten as

$$
\left|\frac{<\chi_{n}(t) \mid \frac{d}{d t^{\prime}} \phi_{n}(t)>e^{-\operatorname{Im}\left[\int_{0}^{t} \omega_{n, m}(s) d s\right]}}{\operatorname{Re}\left[\omega_{n, m}(t)\right]}\right| \ll 1
$$

Now, let us consider the adiabatic evolution of an instantaneous eigenstate of $\mathbf{H}(\mathrm{t})$ under the adiabatic conditions. if the system is initially in state

$$
|\Phi(0)>=U(t=0)| n>=\mid \phi_{n}(0)>,
$$

it will evolve into

$$
\begin{aligned}
\mid \Phi(t)>= & e^{i \gamma_{n}(t)} e^{\frac{1}{i \hbar} \int_{0}^{t} \epsilon_{n}\left(t^{\prime}\right) d t^{\prime}} \mid \phi_{n}(t)> \\
& \equiv e^{i \Omega_{n}(t)} \mid \phi_{n}(t)>
\end{aligned}
$$

where

$$
\Omega_{n}(t)=\gamma_{n}(t)-\frac{1}{\hbar} \int_{0}^{t} \epsilon_{n}\left(t^{\prime}\right) d t^{\prime}
$$

The above eq. (3.8) manifests that the adiabatically- evolving state is always an eigenstate of the instantaneous Hamiltonian $\mathbf{H}(\mathrm{t})$ if the initial state is such state at $\mathrm{t}=0$. This is the quantum adiabatic theorem for $\mathrm{nH}$ quamtum systems, which is quite similar to that for the Hermitian case. It shows the quantum number labelling the quasi-energy level to be an adiabatic invariant. Since $\gamma_{n}(t)$ and $\epsilon_{n}(t)$ are usually not real due to the non-Hermiticity of $\mathbf{H}(\mathrm{t})$, the damping factor $\exp \left[-\operatorname{Im} \Omega_{n}(t)\right]$ causes the adiabatic decay of the state. Especially, when $\epsilon_{n}(t)$ is real, the decay only results from the $\mathrm{nH}$-analog of $\mathrm{BPF}$ and is a purely geometrical effect. In next section an example will be used to illustrate this situation.

When the adiabatic conditions (3.7) are broken, there may appear the transitions from an instantaneous eigenstate $\mid \phi_{n}(0)>$ to others $\mid \phi_{n}(t)>$ for $m \neq n$. From eqs.(3.5) or $(2.5,2.9,2.10)$, we obtaine the transition probabilities

$$
P(n \rightarrow m)=\frac{1}{\hbar^{2}}\left|\int_{0}^{t}<m\right| \mathbf{V}\left(t^{\prime}\right)\left|n>e^{i \int_{0}^{t^{\prime}} \omega_{m, n}(s) d s} d t^{\prime}\right|^{2} e^{-2 \operatorname{Im}[\Omega(t)]}
$$


or

$$
P(n \rightarrow m)=\frac{1}{\hbar^{2}}\left|\int_{0}^{t}<\chi_{m}\left(t^{\prime}\right)\right| \frac{d}{d t^{\prime}} \phi_{n}\left(t^{\prime}\right)>\left.e^{i \int_{0}^{t^{\prime}} \omega_{m, n}(s) d s} d t^{\prime}\right|^{2} e^{-2 \operatorname{Im}[\Omega(t)]} .
$$

Therefore, we reach the statement mentioned in section.2 that the diagonal and offdiagnoal parts respectively describe the adiabatic evolution and the non-adiabatic transitions for the quantum system. Notice that Berry also discussed the geometrical phase effect in non-adiabatic transition in connection with the non-Hermiticity due to the complexization of time [22].

\section{Example:Generalized Force Hamornic Oscillator}

Now, let us apply the above general analysis to a simple model - the generalized forced harmonic oscillator. The time-dependent Hamiltonian for this model is

$$
\mathbf{H}(t)=\mathbf{H}[\alpha, \beta]=\hbar \omega\left(a^{\dagger} a+\beta a^{\dagger}+\alpha a\right)
$$

where $\alpha=\alpha(t)$ and $\beta=\beta(t)$ are slowly-changing complex parameters, the constant $\omega$ is real , $a$ and $a^{\dagger}$ are respectively the creation and annihilation operators for a boson state, which satisfy

$$
\left[a, a^{\dagger}\right]=1
$$

For the usual forced harmonic oscillator (FHO), there is constraint $\alpha=\beta^{*}$ and the

corresponding Hamiltonian is Hermitian. However, we make here a generalization to remove this constraint so that the Hamiltonian (4.10) is non-Hermitian.

Using the translated boson operators

$$
A(\alpha)^{\dagger}=a^{\dagger}+\alpha, \quad A(\beta)=a+\beta
$$

obeying the same boson commutation relation as the above

$$
\left[A(\beta), A(\alpha)^{\dagger}\right]=1
$$

we rewrite down the Hamiltonian (4.1) as

$$
\mathbf{H}(t)=\mathbf{H}[\alpha, \beta]=\hbar \omega\left[A(\alpha)^{\dagger} A(\beta)-\alpha \beta\right] .
$$


Based on the above expression for $\mathbf{H}(\mathrm{t})$, we immediately obtain the instantaneous eigenstates of $\mathbf{H}(t)=\mathbf{H}[\alpha, \beta]$

$$
\begin{gathered}
\left|\phi_{n}(t)>=\right| \phi_{n}[\alpha, \beta]>=\mid n[\alpha, \beta]> \\
=\frac{1}{\sqrt{n !}}\left[A(\alpha)^{\dagger}\right]^{n} \mid 0(\beta)>
\end{gathered}
$$

with the eigenvalues

$$
\epsilon_{n}(t)=\epsilon_{n}[\alpha, \beta]=(n-\alpha \beta) \hbar \omega
$$

Here, the translated vacuum state $\mid 0(\beta)>$ obeys

$$
A(\beta) \mid 0(\beta)>=0, \text { or } \quad a|0(\beta)>=-\beta| 0(\beta)>,
$$

that is to say, $\mid 0(\beta)>$ is a coherent state

$$
\left|0(\beta)>=C_{\alpha, \beta} e^{-\beta a^{\dagger}}\right| 0>,
$$

where $\mid 0>$ is the usual vacuum state satisfying $a \mid 0>=0$ and $C_{\alpha, \beta}$ is to be determined by the biorthonormal conditions . According to the above analysis, we can finally write the explicit form of $\mid \phi_{n}(t)>$

$$
\left|\phi_{n}(t)>=C_{\alpha, \beta} \sum_{k=0}^{n} \frac{n ! \beta^{n-k}}{(n-k) ! \sqrt{k !}} e^{-\alpha a^{\dagger}}\right| k>,
$$

where

$$
\left|k>=\frac{1}{\sqrt{k !}}\left[a^{\dagger}\right]^{k}\right| 0>
$$

is the $\mathrm{k}$ boson state.

Noticing

$$
\mathbf{H}[\alpha, \beta]^{\dagger}=\mathbf{H}\left[\beta^{*}, \alpha^{*}\right]
$$

we easily obtain the dual vector $\mid \chi_{n}(t)>$ to $\mid \phi_{n}(t)>$ :

$$
\left|\chi_{n}(t)>=\right| \chi_{n}[\alpha, \beta]>=\mid n\left[\beta^{*}, \alpha^{*}\right]>
$$

as the eigenvectors of $\mathbf{H}[\alpha, \beta]^{\dagger}$ with the corresponding eigenvalue $\epsilon_{n}\left[\beta^{*}, \alpha^{*}\right]$. Then, the biorthonormal conditions

$$
<\chi_{n}(t) \mid \phi_{m}(t)>=\delta_{m, n}
$$


define the biorthonormalization coeeficient

$$
C_{\alpha, \beta}=e^{-\frac{1}{2} \alpha \beta}
$$

Taking into acount the translation transformation

$$
\begin{gathered}
e^{\xi a} a^{\dagger} e^{-\xi a}=a^{\dagger}+\xi, \\
e^{\xi a^{\dagger}} a e^{-\xi a^{\dagger}}=a-\xi, \\
\xi \in \text { complex field } C
\end{gathered}
$$

we get the $\mathrm{U}(\mathrm{t})$-operator expression of the biorthonormal basis $\left\{\left|\phi_{m}(t)>,\right| \chi_{n}(t)>\right\}$

$$
\begin{gathered}
\left|\phi_{n}(t)>=\right| \phi_{n}[\mathbf{R}]>=U(t) \mid n>, \\
\left|\chi_{n}(t)>=\right| \chi_{n}[\mathbf{R}]>=\left[U(t)^{-1}\right]^{\dagger} \mid n>,
\end{gathered}
$$

where the $\mathrm{U}(\mathrm{t})$-operator

$$
U(t)=U[\alpha, \beta]=e^{-\frac{1}{2} \alpha \beta} e^{-\beta a^{\dagger}} e^{-\alpha a}
$$

diagonalizes the Hamiltonian $\mathbf{H}(\mathrm{t})$, i.e.,

$$
U(t)^{-1} \mathbf{H}(t) U(t)=\hbar \omega a^{\dagger} a
$$

Now, let us discuss the evolution governed by the nH Hamiltonian (4.1). To this end, we first calculate

$$
\begin{aligned}
<\chi_{m}(t) \mid \frac{d}{d t} \phi_{n}(t)> & =\sqrt{n} \dot{\alpha} \delta_{m, n-1}-\sqrt{n+1} \dot{\beta} \delta_{m, n+1} \\
+ & \frac{1}{2}[\alpha \dot{\beta}-\beta \dot{\alpha}] \delta_{m, n}
\end{aligned}
$$

Then, we obtain the $\mathrm{nH}$ analog of $\mathrm{BPF}$

$$
\begin{aligned}
e^{i \gamma_{n}(t)} & \equiv e^{i \gamma(t)}=\exp \left\{\frac{i}{2} \int_{0}^{t}[\alpha \dot{\beta}-\beta \dot{\alpha}]\left(t^{\prime}\right) d t^{\prime}\right\} \\
& =\exp \left\{\frac{i}{2} \int_{C ; \mathbf{R}(0)}^{\mathbf{R}(t)}[\alpha d \beta-\beta d \alpha]\right\}
\end{aligned}
$$

where $\mathrm{C}$ is a curve $\{\mathbf{R}(\mathrm{t})\}$ in a four dimensional parameter manifold

$$
\mathbf{M}:\left\{\mathbf{R}=(\alpha, \beta)=\left(\alpha_{1}, \alpha_{2}, \beta_{1}, \beta_{2}\right) \mid \alpha=\alpha_{1}+i \alpha_{2}, \beta=\beta_{1}+i \beta_{2}\right\}
$$


where $\alpha_{1}, \alpha_{2}, \beta_{1}$ and $\beta_{2}$ are real. Because the factor $e^{i \gamma_{n}(t)}$ is independent of the quantum number $\mathrm{n}$, the adiabatic evolution

$$
\begin{gathered}
\left|\Phi(t)>=e^{i \gamma(t)} \sum_{n=1}^{N}<\chi_{n}(0)\right| \Psi(0)>e^{-i n \omega t} \mid \phi_{n}(t)> \\
\equiv e^{-\Gamma(t)} e^{i \Theta(t)} \sum_{n=1}^{N}<\chi_{n}(0)\left|\Psi(0)>e^{-i n \omega t}\right| \phi_{n}(t)
\end{gathered}
$$

is accompanied with a global geometrical factor with the oscillating part $e^{i \Theta(t)}$ :

$$
\Theta(t)=\operatorname{Re}\left[\gamma_{n}(t)\right]=\frac{i}{2} \int_{0}^{t}\left[\beta_{1} \dot{\alpha}_{2}+\beta_{2} \dot{\alpha}_{1}-\alpha_{2} \dot{\beta}_{1}-\alpha_{1} \dot{\beta}_{2}\right]\left(t^{\prime}\right) d t^{\prime}
$$

and the damping part $e^{-\Gamma(t)}$ :

$$
\Gamma(t)=\operatorname{Im}\left[\gamma_{n}(t)\right]=\frac{i}{2} \int_{0}^{t}\left[\beta_{2} \dot{\alpha}_{2}-\beta_{1} \dot{\alpha}_{1}-\alpha_{2} \dot{\beta}_{2}+\alpha_{1} \dot{\beta}_{1}\right]\left(t^{\prime}\right) d t^{\prime}
$$

Notice that, when $\alpha(t)=\beta(t)^{*}$, the BPF of coherent state for FHO in ref.[23] is given once again as a special case of eq.(4.9).

For the case with large quantum number $\mathrm{n}$ or rapidly-changing parameters $\alpha(t)$ and $\beta(t)$, the adiabatic conditions

$$
\frac{\sqrt{n}|\dot{\alpha}(t)|}{\omega} e^{-\Gamma(t)} \ll 1 ; \frac{\sqrt{n+1}|\dot{\beta}(t)|}{\omega} e^{-\Gamma(t)} \ll 1
$$

do not hold and we need to consider the non-adiabatic effects caused by the first order approximation at least. If the system is initially in a state $\mid \phi_{k}(0)>$, the initial conditions

$$
C_{n}^{0}(0)=\delta_{n, k} \quad, \quad C_{n}^{l}(0)=0, \text { for } l \geq 1
$$

leads to the first order approximate solution

$$
\begin{gathered}
\left|\Psi^{1}(t)>=\sqrt{k} e^{-\Gamma(t)} e^{i[\Theta(t)-(k-1) \omega t]} \int_{0}^{t} \dot{\alpha}\left(t^{\prime}\right) e^{\left.-i \omega t^{\prime}\right]} d t^{\prime}\right| \phi_{k-1}(t)> \\
+\sqrt{k+1} e^{-\Gamma(t)} e^{i[\Theta(t)-(k+1) \omega t]} \int_{0}^{t} \dot{\beta}\left(t^{\prime}\right) e^{i \omega t^{\prime}} d t^{\prime} \mid \phi_{k+1}(t)>
\end{gathered}
$$

The probabilities of the transition from $\mid \phi_{n}(0)>$ to $\mid \phi_{n}(t)>(\mathrm{n}=\mathrm{k}-1, \mathrm{k}+1)$ are $P(k \rightarrow n)=k e^{-2 \Gamma(t)}\left|\int_{0}^{t} \dot{\alpha}\left(t^{\prime}\right) e^{-i \omega t^{\prime}} d t^{\prime}\right|^{2} \delta_{n, k-1}+(k+1) e^{-2 \Gamma(t)}\left|\int_{0}^{t} \dot{\beta}\left(t^{\prime}\right) e^{\left.i \omega t^{\prime}\right]} d t\right|^{2} \delta_{n, k+1}$. 
Obviously, the selection rule for such transition is

$$
\Delta \mathrm{n}=+1 \quad \text { or } \quad-1
$$

under the first order approximation.

\section{The nH-Analog of BPF and Holonomy in Dual Line Fiber Bundles}

For Hermitian quantum system, Simon recognized that the BPF is precisely the holonomy in a Hermitian line bundle defined by the adiabatic evolution. The adiabatic evolution can be interpreted as a parallel translation in such bundle [24]. Now, a question naturally arises for the non-Hermitian case: what is a geometrical interpretation of the $\mathrm{nH}$ analog of BPF? The answer will be given in this section.

Let $\mathbf{M}$ be the parameter manifold formed by the parameters $\mathbf{R}=\left(R_{1}, R_{2}, \ldots, R_{L}\right)$ . A line bundle defined by the $\mathrm{nH}$ Hamiltonian $\mathbf{H}[\mathbf{R}]$ is

$$
\mathbf{F}_{n}=\left\{\left(\mathbf{R}, \mid \sigma_{n}[\mathbf{R}]>\right)|\mathbf{H}[\mathbf{R}]| \sigma_{n}[\mathbf{R}]>=\epsilon_{n}[\mathbf{R}] \mid \sigma_{n}[\mathbf{R}]>, \mathbf{R} \in \mathbf{M}\right\} .
$$

Its fiber space is a one-dimensional linear space

$$
\mathbf{V}_{\mathbf{n}}:\left\{\left|\sigma_{n}[\mathbf{R}]>=e^{i \theta[\mathbf{R}] \mid} \phi_{n}[\mathbf{R}]>\right| \theta[\mathbf{R}] \text { is a real functions depending on } \mathbf{R}\right\}
$$

Over the same base manifold $\mathbf{M}$, the dual bundel $\mathbf{F}_{n}^{*}$ is defined by

$$
\mathbf{F}_{n}^{*}=\left\{\left(\mathbf{R}, \mid \sigma_{n}^{*}[\mathbf{R}]>\right)\left|\mathbf{H}[\mathbf{R}]^{\dagger}\right| \sigma_{n}^{*}[\mathbf{R}]>=\epsilon_{n}^{*}[\mathbf{R}] \mid \sigma_{n}^{*}[\mathbf{R}]>, \mathbf{R} \in \mathbf{M}\right\} .
$$

where the fiber space

$$
\mathbf{V}_{\mathbf{n}}^{*}:\left\{\left|\sigma_{n}^{*}[\mathbf{R}]>=e^{i \theta^{\prime}[\mathbf{R}]}\right| \chi_{n}[\mathbf{R}]>\mid \theta^{\prime}[\mathbf{R}] \text { is a real functions depending on } \mathbf{R}\right\}
$$

is the dual space of $\mathbf{V}_{\mathbf{n}}$ and $\mid \chi_{n}[\mathbf{R}]>$ is the dual elemet of $\mid \phi_{n}[\mathbf{R}]>$, i.e.,

$$
<\chi_{n}[\mathbf{R}] \mid \phi_{n}[\mathbf{R}]>=\delta_{m, n} .
$$

Since the quantum number $n$ labelling an eigenstate $\mid \phi_{n}[\mathbf{R}]>$ of the instantaneous Hamiltonian $\mathbf{H}[\mathbf{R}]$ is an adiabatic invariant, we can assume that

$$
\left|\sigma_{n}(t)>=\right| \sigma_{n}[\mathbf{R}]>=C_{n}[\mathbf{R}] \mid \phi_{n}[\mathbf{R}]>
$$


is an evolution state in adiabatic case. Now, let us show that the holonomy group elements of $\mathbf{F}_{n}\left(\mathbf{F}_{n}^{*}\right)$ is the $\mathrm{nH}$ analog of BPF for the adiabatic evolution while $\left\{\left|\sigma_{n}(t)>\right| t \in[0, T]\right\}\left(\left\{\left|\sigma *_{n}(t)>\right| t \in[0, T]\right\}\right)$, as a curve in $\mathbf{F}_{n}\left(\mathbf{F}_{n}^{*}\right)$, is a horizontal lift of the curve $C:\{\mathbf{R}(t) \mid t \in[0, T]\}$ on base manifold $\mathbf{M}$. To this end we consider a decomposition of a tangent vector

$$
\frac{d}{d t}\left|\sigma_{n}(t)>=\frac{d_{v}}{d t}\right| \sigma_{n}(t)>+\frac{d_{h}}{d t} \mid \sigma_{n}(t)>
$$

where the vertical part along the fiber is

$$
\begin{gathered}
\frac{d_{v}}{d t}\left|\sigma_{n}(t)>=<\chi_{n}[\mathbf{R}]\right| \frac{d}{d t}\left|\sigma_{n}[\mathbf{R}]>\right| \phi_{n}[\mathbf{R}]> \\
=\left(\frac{d}{d t} C_{n}[\mathbf{R}]+<\chi_{n}\left[\mathbf{R}\left|\frac{d}{d t}\right| \phi_{n}[\mathbf{R}]>C_{n}[\mathbf{R}]\right) \mid \phi_{n}[\mathbf{R}]>\right.
\end{gathered}
$$

and the horizontal part orthogonal to the fiber is

$$
\frac{d_{h}}{d t}\left|\sigma_{n}(t)>=\sum_{m \neq n}<\chi_{m}[\mathbf{R}]\right| \frac{d}{d t}\left|\sigma_{n}[\mathbf{R}]>\right| \phi_{m}[\mathbf{R}]>.
$$

A parallel-translation implies

$$
<\chi_{n}\left[\mathbf{R}\left|\frac{d_{v}}{d t}\right| \sigma_{n}(t)>=0,\right.
$$

which results in a one-form equation

$$
d C_{n}[\mathbf{R}]+<\chi_{n}\left[\mathbf{R} \mid d \phi_{n}[\mathbf{R}]>C_{n}[\mathbf{R}]=0 .\right.
$$

Its solution

$$
C_{n}(t)=C_{n}[\mathbf{R}(\mathbf{t})]=e^{i \gamma_{n}(t)}
$$

just gives the $\mathrm{nH}$ analog of $\mathrm{BPF}$ again.

For a cyclic evolution that $\mathbf{R}(0)=\mathbf{R}(t)$ and $\mathrm{C}$ is a closed curve, the complex phase $\gamma_{n}(t)$ in the $\mathrm{nH}$ analog of BPF can be expressed as an closed path integration

$$
\gamma_{n}(T)=\gamma_{n}[C]=\oint_{C} \mathbf{A}_{\mathbf{n}}[\mathbf{R}]
$$

of a complex potential one-form

$$
\mathbf{A}_{\mathbf{n}}[\mathbf{R}]=i<\chi_{n}[\mathbf{R}] \mid d \phi_{n}[\mathbf{R}]>
$$


Similarly, on dual bundel $\mathbf{F}_{n}^{*}$, the parallell-translation condition

$$
<\phi_{n}[\mathbf{R}]\left|\frac{d_{v}}{d t}\right| \sigma *_{n}(t)>=0
$$

results in the dual $\mathrm{nH}$ analog of $\mathrm{BPF}$ with the geometric phase

$$
\bar{\gamma}_{n}(t)=i \int_{0}^{t}<\phi_{n}\left[\mathbf{R}\left(t^{\prime}\right)\right] \mid \frac{d}{d t^{\prime}} \chi_{n}\left[\mathbf{R}\left(t^{\prime}\right)\right]>d t^{\prime} .
$$

Obviously, the $\mathrm{nH}$ analog $\exp \left[\gamma_{n}(t)\right]$ and its dual $\exp \left[\bar{\gamma}_{n}(t)\right]$ occuring in the adiabatic evolution are holonomy group elements on the line bundel $\mathbf{F}_{n}$ and its dual $\mathbf{F}_{n}^{*}$ respectively. Except for the effect of dynamical factors

$$
f(t)=e^{\frac{1}{i \hbar} \int_{0}^{t} \epsilon\left[\mathbf{R}\left(t^{\prime}\right)\right] d t^{\prime}}, \bar{f}(t)=e^{\frac{1}{\hbar \hbar} \int_{0}^{t} \epsilon\left[\mathbf{R}\left(t^{\prime}\right)\right]^{*} d t^{\prime}}
$$

the adiabatic effect in quantum evolution are equivalent to the parallel-translation on the line bundels $\mathbf{F}_{n}$ and its dual $\mathbf{F}_{n}^{*}$. This is a circumstance similar to that for the Hermitian quantum system.

\section{Acknowledgements}

This work is supported by Cha Chi- Ming fellowship through the CEEC in State University of New York at Stony Brook. The author is also supported in part by the NFS of China and The Fok Ying-Tung Education Foundation through Northeast Normal University. 


\section{References}

1. M.V.Berry, Proc.R.Soc.Lond.,A392 (1984),45; for a complete review, see Geometric Phases in Physics, ed, by A.Shapere and F.Wilczek, World Scientific,Sigapore, 1989.

2. M.V.Berry, Proc.R.Soc.Lond.,A414 (1987),31.

3. N.Nakagawa, Ann.Phys., 179(1987),145.

4. C.P.Sun, J.Phys.A,21(1988),1585; High Energy Phys.Nucl.Phys.,12(1988), 351;

Phys.Rev.D, 41(1990),1318.

5. C.M.Bender and N.Papanicolaou,J.Phys.France, 49(1988),561; 1493.

6. C.P.Sun,Phys.Rev.D, 38(1988),2908.

7. C.P.Sun and M.L.Ge,Phys.Rev.D, 41(1990),1349; Commun.Theor.Phys., 13(1990),63;

C.P.Sun and L.Z.Zhang, Commun.Theor.Phys.12(1989) 479.

8. C.P.Sun, Chinese Phys.Lett.,6(19989),481.

9. Z.Y.Wu,Phys.Rev.A ,40(1989) 2184; 6852.

10. B.L.Markovski, in Topological Phases in Quantum Theory, ed. by B.L.Markovsk and S.I.Vinitsky, World Scientific,Sigapore, 1989.

11. S.I.Vinitsky, V.L.Derbov, V.N.Dubovski, B.L.Markovskiand Yu.P.Stepanovski,Sov.Phys.Usp. $33(1990), 403$.

12. C.P.Sun ,L.wang and M.L.Ge, Commun.Theor.Phys.,15(1991),427.

13. V.Fock and K.Krylov,Zh.Eksp.Fiz.17(1947),93.

14. E.Divies, Quantum Theory of Open system, Academic, New York, 1976.

15. P.Exner, Open Quantum system and Feynman Integrals, Reidel, Dordrecht, 1985. 
16. F.M.Faysal and J.V.Moloney, J.Phys.B, 14(19820, 3603.

17. A.Siegman, Optics Commu.,31(1979),369.

18. H.C.Baker,Phys.Rev.Lett.,50(1983),1579; Phys.Rev.A,30(1984),773.

19. G.Dattoli,A.Torre and R.Mignani, Phys.Rev.A,42(1990),1476; and refs. therein.

20. C.Miniatura, C .Sire,J.Baudon and J.Bellissad, Europ.Phs.Lett.,13(19900, 199.

21. G.Dattoli, R.Mignani and A.Torre, J.Phys.A,23(1990),5795..

22. M.V.Berry, Proc.R.Soc.Lond.,A,430,405;429(1990),61.

23. S.Chaturved,M.S.Sriram and V.Srinivasan, J.Phys.A,20(1987),L1071.

24. B.Simon,Phys.Rev.Lett.,51(1983),2167. 